\title{
Análisis de sistemas integrados de gestión bibliotecaria de acceso abierto: examen de tres casos concretos
}

\author{
Paula Ramón Olivert \\ Graduada en Información y Documentación
}

Recibido el

\begin{abstract}
Resumen
El crecimiento de las comunidades internautas y la popularidad del código libre han propiciado el auge de la distribución de sistemas integrados de gestión bibliotecaria de acceso abierto. En el presente trabajo se realiza un análisis sobre estos programas actualmente disponibles en la red. Para ello, se ha ejecutado una intensa búsqueda en internet con el fin de recuperar los sistemas integrados de acceso abierto más habituales, más conocidos, utilizados y con mayor visibilidad. De entre ellos se seleccionaron los software Biblio, BiblioteQ y CaMPI, ya que cumplían con los requisitos de completar satisfactoriamente el proceso de instalación, y abarcar las funcionalidades necesarias para automatizar una biblioteca. Estos sistemas se examinaron pormenorizadamente, con el fin de conocer en profundidad su funcionamiento, sus módulos y características más significativas para la utilización, como pueden ser el idioma o su manejabilidad. Se puede concluir que hoy en día los sistemas integrados de gestión bibliotecaria de acceso abierto se presentan al mercado de la gestión de la información como una alternativa sólida a los softwares privados o propietarios, contando además con una gran perspectiva de futuro.
\end{abstract}

\section{Palabras clave}

Acceso abierto, automatización de bibliotecas, Biblio, BiblioteQ, CaMPI, programas de gestión bibliotecaria, sistemas de gestión bibliotecaria, sistemas integrados de gestión bibliotecaria, SIGB.

\section{Title}

Analysis of open source integrated library management systems: a review of three specific cases.

\begin{abstract}
The growth of Internet communities and the free code popularity have favoured the rise of the distribution of open access integrated library management systems. In the present paper, an analysis concerning these currently available programmes on the net is carried out. For this, an intense search on the Internet has been executed in order to recover the most current, well-known, used and visible open access integrated systems. Among them, Biblio, BiblioteQ and CaMPI software were selected, since they met the requirements for completing successfully the installation process, and taking on the necessary functionalities in order to automate a library. These systems were examined in detail, with the intention of obtaining an in-depth knowledge of their functioning, modules and
\end{abstract}


most significant characteristics for their use, such as the language or their handiness. One may conclude that today the open access integrated library management systems are presented as a sound alternative to private or proprietary software, also relying on a great future outlook.

\section{Keywords}

Open access, library automation, Biblio, BiblioteQ, CaMPI, library management programmes, library management systems, integrated library management systems, ILS.

\section{1.- INTRODUCCIÓN}

La investigacióEn el contexto bibliotecario y documental, Luis A. García Melero y Ernesto García Camarero definen la automatización de unidades de información como:

"El conjunto organizado de recursos humanos que utilizan dispositivos y programas informáticos, adecuados a la naturaleza de los datos, para realizar los procesos y facilitar los servicios que permiten alcanzar el objetivo de la biblioteca." (García Melero, L.A. y García Camarero, E., 1999).

En el año 1984, Lucy A. Tedd mencionaba un informe del año 1972 donde, entre otros, se dan los siguientes argumentos para el desarrollo de sistemas informáticos bibliotecarios: "Proporcionar un servicio de mayor calidad a menor o igual coste" y "ofrecer un mayor número de servicios a bajo coste". Es decir, en el mundo de la información ya no se polemiza sobre si "automatizar o no”, sino que, como señala Dennis Reynolds (1985), se estudian los factores e influencias que determinarán la adopción de una determinada tecnología por las bibliotecas.

\section{1.- Sistemas Integrados de Gestión Bibliotecaria (SIGB)}

Los sistemas integrados de gestión bibliotecaria, desde ahora SIGB, son los programas que gestionan la automatización de las bibliotecas. Félix de Moya (1995) define los sistemas de información automáticos (SIA) "en base a sus elementos constituyentes y a su objeto", es decir, "como un subconjunto del 
sistema de información real”. Por lo tanto, un SIGB es un programa compuesto de distintas aplicaciones interrelacionadas entre sí, conocidas como módulos, que comparten las mismas bases de datos evitando de este modo posibles redundancias en la información y aumentando la eficacia del sistema.

Los SIGB suelen estar desarrollados por empresas privadas, los hay realizados por un centro concreto para uso propio, y también se han creado sistemas de acceso abierto.

En el año 2008, Tristan Müller explicó que para muchas bibliotecas es financieramente difícil adquirir un sistema propietario, ya que el coste medio de base puede oscilar entre los 20.000 y 100.000 \$CAN (aproximadamente, 13.000 $-65.500 €)$ y de 2.000 a 10.000 CAN $(1.300-6.500 €)$ para la licencia anual. En este punto, también se puede considerar la opción de desarrollar un sistema propio, pero García Melero y García Camarero (1999) advertían al respecto que se debe tener una infraestructura adecuada y una considerable dedicación humana y técnica, lo cual puede suponer al final un mayor coste que la adquisición de un sistema comercial.

Marshall Breeding (2010) destaca que el tamaño del mercado de la automatización de bibliotecas se estima en unos $\$ 630$ millones (unos 455 millones de euros). Posteriormente, el mismo autor recalca que teniendo en cuenta los presupuestos de las bibliotecas, el mantenimiento de los SIGB propietarios era inviable, de ahí que consideraran la migración a otros programas (Breeding 2011). Müller (2008) explica que la participación en las comunidades libres de internet junto a las dificultades económicas ha favorecido la emergencia de nuevos SIGB de licencia libre, considerados una solución viable.

En este trabajo se va a englobar dentro del acceso abierto a los programas gratuitos junto a los de código abierto. Cabe recordar que no es lo mismo un software propietario con acceso libre, que el usuario puede utilizar gratuitamente pero sin tener acceso al código; que un software de código abierto o "libre", que también es gratuito pero que sí permite al usuario la modificación del código del programa.

\section{2.- Justificación del uso de SIGB de acceso abierto frente a los SIGB propietarios.}

La actual situación económica de España lleva a plantear si se puede hacer 
frente de manera realista al abanico de costes que supone en una biblioteca de cualquier ámbito la implementación de un SIGB privado o la creación de uno para su uso particular. Teniendo en cuenta lo reducido de los presupuestos dedicados a Cultura por parte de la Administración Pública, la incorporación o la adquisición de un software integrado de acceso abierto se presenta como una buena alternativa.

Si se suma la necesidad de automatización en las bibliotecas y centros a la expansión de los programas libres, así como las condiciones económicas que dificultan el acceso al software privado y de creación propia, está plenamente justificada la realización de un trabajo centrado en el análisis de los SIGB de acceso abierto. Este trabajo permitirá tener una visión global de la amplísima y creciente oferta disponible en la actualidad en relación con los SIGB de acceso abierto, así como conocer las características de los mismos analíticamente. Este estudio pretende ser un punto de partida para todas las instituciones, centros o entidades singulares o plurales que necesiten realizar un proceso de automatización, guiándoles hacia el que más se adecue a sus necesidades.

\section{2.- METODOLOGÍA}

Se comenzó realizando una búsqueda bibliográfica y documental de carácter generalista, con el fin de afianzar los conocimientos adquiridos acerca de la automatización de bibliotecas y los distintos tipos de software integrados de gestión bibliotecaria, para poder diferenciar correctamente entre los SIGB propietarios y los de acceso abierto, o los de código libre.

Las fuentes de información consultadas a tal fin fueron $\mathrm{El}$ profesional de la información, la Enciclopedia Larousse, la Enciclopedia Británica, la Enciclopedia Espasa, la Gran enciclopèdia catalana, Mienciclo Universal y Wikipedia. El buscador empleado fue Google. Simultáneamente, se fueron anotando las menciones a diferentes programas que la red nombraba e identificaba como SIGB de acceso abierto, pero que aún no se había verificado y contrastado si realmente lo eran.

La estrategia de búsqueda comenzó con la consulta del Tesauro de Biblioteconomía y Documentación del CSIC, con el objetivo de recabar las palabras clave que se utilizarían en las consultas en las diferentes fuentes de información. A continuación, se sumaron otras palabras sinónimas a las anteriores, y sus equivalentes en inglés: 
- Acceso abierto / Open Access / Open Source

- Biblioteca / Bibliotecario / Library / Librarian

- Código abierto / Open Code

- Gestión bibliotecaria / Library Management

- Sistema integrado / SI / Integrated System / IS

- Sistema integrado de gestión bibliotecaria / SIGB / Integrated Library System / ILS

- $\quad$ Programa libre / Software libre / Free software

En las búsquedas se emplearon operadores booleanos, truncamientos y diferentes combinaciones entre las palabras clave extraídas del tesauro, las sinónimas y en inglés.

En la primera fase de la búsqueda, se recopilaron las menciones de diferentes SIGB de acceso abierto en Google, ampliando después a otros buscadores como Ask, Bing, Hakia y Yahoo. Estos últimos aportaron nuevos resultados a los recuperados por Google, y de este modo se logró recopilar un listado de 33 menciones a programas diferentes.

La segunda fase de la búsqueda consistió en la indagación en el buscador de artículos Google Académico, junto a:

- Las bases de datos Dialnet, Lisa, Lista, Redalyc y Scopus.

- Los directorios Doaj, Hispana y OpenDoar.

- Los repositorios Roar, Teseo y Tdr.

- El recolector de repositorios Recolecta.

Tras esta exhaustiva búsqueda se recuperaron otros 13 nombres de programas que en la red figuraban como SIGB de acceso abierto, dando un listado total de 47. Si bien es cierto que posiblemente exista más software en la red, en este trabajo han sido recuperados los más habituales, más conocidos, utilizados y que tienen una mayor visibilidad. En los resultados se desarrolla pormenorizadamente la verificación de si estos programas que la red identifica como SIGB de acceso abierto realmente lo son, comprobando que en algunos casos no lo fueron.

Estos son los 47 programas que se hallaron en la red mencionados como SIGB libre, de acceso abierto o de código abierto: 
- $\mathrm{ABCD}$

- Aguapey

- Avanti MicroLCS

- BiblioDOC

- Biblio

- BiblioteQ

- Biblivre

- CaMPI

- Catalis

- CDS/ISIS

- E-Library

- Emilda

- EverGreen

- GBwin32

- GLIBMS (GNU Library Management System)

- Gnuteca

- Greenstone Digital Library Software

- Infocid

- Invenio

- Janium

- Karuna

- Koha./Kobli

- Learning Access ILS
- Microisis/BIREME

- Millennium

- MiniSOPULI

- NewGenLib

- Next-L Enju

- Odilo

- Opals

- OpenBiblio

- OpenBook

- OpenMarcoPolo

- OpenOpac

- OtomiGenX

- PCAm8.0

- PhpMyBibli (PMB)

- PHPMyBiblio

- PHPMyLibrary

- SABINI

- Senayan Library Management System (SLiMS)

- Techlib

- Unicorn

- Utls

- Web Librarian

- Weblis

- WorldCat

Uno de los criterios empleados en el momento de discriminar los softwares fue la capacidad de utilización de los mismos por una persona con conocimientos informáticos medios dentro el ámbito universitario. El presente trabajo se ha realizado íntegramente en un ordenador portátil Acer Aspire One y con ADSL $50 \mathrm{Mb}$ de ONO. Un ordenador con una potencia o un ancho de banda distinto puede afectar al funcionamiento de los programas.

\section{3.- RESULTADOS}

Los sistemas que aparecen en el listado de los 47 programas mencionados como SIGB de acceso abierto con más visibilidad en internet se verificaron por orden alfabético. Una vez concluido el análisis, se ha decidido mostrar los resultados agrupados por categorías, con el fin de condensar la información y evitar redundancias innecesarias. Estas categorías son excluyentes entre sí, y 
se establecieron en base a los distintos tipos de resultados obtenidos de la verificación, que fueron:

- 3 SIGB verificados con instalación satisfactoria.

Se seleccionaron estos programas para profundizar en su análisis porque cumplían con los siguientes requisitos: ser un SIGB, de acceso abierto, disponible en la web, y de fácil instalación y manejo.

- Biblio

- BiblioteQ

- CaMPI

- 4 SIGB con mayor visibilidad en la red.

Son los SIGB de acceso abierto, y en estos casos también de código libre, con mayor visibilidad en la red. Cuentan con comunidades internautas muy activas que los desarrollan y mantienen actualizados con frecuencia, lo que aumenta asimismo la repercusión de estos programas en la web. Requieren la realización de cursos de formación de pago.

- Koha

- Evergreen

- Opals

- PhpMyBibli (PMB)

- 14 SIGB con un proceso de instalación no completado.

$\mathrm{Su}$ instalación no se completó por varios problemas, como por ejemplo la ausencia de un archivo autoejecutable ".exe”; la obligatoriedad de registrarse como usuario y disponer de contraseña pero sin explicar cómo efectuar ese registro; o la falta de programas adicionales no proporcionados por el instalador, no disponibles en la red o que requieren de unos conocimientos informáticos avanzados para su instalación. 
Biblivre

Catalis

E-library

Emilda

Invenio

MiniSOPULI

NewGenLib

Next-L Enju
OpenBiblio

OpenOpac

OtomiGenX

PhpMyLibrary

Senayan Library Manage-

ment System (SLiMS)

WebLibrarian

- 26 SIGB no disponibles actualmente en la red.

En ocasiones bajo el nombre del programa se ha hallado el sitio web de una empresa o un software que no corresponde con un SIGB; había una incompatibilidad con los sistemas operativos actuales; o el sitio web oficial ya no existía, y no se había encontrado otro sitio. También cabe destacar que pese a mencionarse como gratuitos, había programas que requerían desembolso económico.

ABCD
Aguapey
Avanti microlcs- 1.0 beta 4
BiblioDOC
CDS/ ISIS
GBwin32
GNU library management
$\quad$ system
Gnuteca
Greenstone Digital Library
$\quad$ Software
Infocid
Janium

Karuna
Learning Access ILS
Microisis/ BIREME
Millennium
Odilo
OpenBook
OpenMarcoPolo
PCAm8.0
PHPMyBiblio
Sabini
Techlib
Unicorn
Utils

Los programas verificados con instalación satisfactoria (Biblio, BiblioteQ y CaMPI), una vez implementados en el ordenador, se analizaron para así esclarecer sus principales características. Para poder compararlos entre sí pese a sus diferencias, se establecieron las mismas categorías para todos, como se puede apreciar en esta tabla de elaboración propia: 
MEI, II, Vol. 6, no 10, pág. 37

\begin{tabular}{|c|c|c|c|}
\hline Característica & Biblio & BiblioteQ & CaMPI \\
\hline Idiomas & $\begin{array}{l}\text { Castellano } \\
\text { Gallego } \\
\text { Inglés }\end{array}$ & $\begin{array}{l}\text { Checo } \\
\text { Holandés de Bélgica } \\
\text { Holandés de Holanda } \\
\text { Inglés } \\
\text { Francés } \\
\text { Alemán } \\
\text { Griego }\end{array}$ & Español latino \\
\hline Instalación & $\begin{array}{l}\text { Archivo en el sitio } \\
\text { web oficial } \\
\text { Muy sencilla, in- } \\
\text { tuitiva, rápida } \\
\text { No necesita inter- } \\
\text { net para funcio- } \\
\text { nar }\end{array}$ & $\begin{array}{l}\text { Archivo en Source- } \\
\text { forge } \\
\text { Muy sencilla, intuiti- } \\
\text { va, rápida } \\
\text { No necesita internet } \\
\text { para funcionar }\end{array}$ & $\begin{array}{l}\text { Archivo en el sitio } \\
\text { web oficial } \\
\text { Muy sencilla, intuiti- } \\
\text { va, rápida } \\
\text { Necesita internet para } \\
\text { funcionar }\end{array}$ \\
\hline Guía de uso & $\begin{array}{l}\text { Disponible } \\
\text { Explica y usa imá- } \\
\text { genes } \\
\text { En castellano }\end{array}$ & No disponible & $\begin{array}{l}\text { Disponible } \\
\text { Explica y usa imáge- } \\
\text { nes } \\
\text { En español latino }\end{array}$ \\
\hline Interfaz & $\begin{array}{l}\text { Anticuada } \\
\text { Intuitiva } \\
\text { Con pocos iconos } \\
\text { Icono con dibujo y } \\
\text { texto }\end{array}$ & $\begin{array}{l}\text { Moderna } \\
\text { No muy intuitiva } \\
\text { Pocos iconos } \\
\text { Icono con dibujo }\end{array}$ & $\begin{array}{l}\text { Anticuada } \\
\text { Intuitiva } \\
\text { Pocas opciones } \\
\text { Botones con texto }\end{array}$ \\
\hline Manejabilidad & $\begin{array}{l}\text { Nivel de informáti- } \\
\text { ca básico } \\
\text { No hay que saber } \\
\text { catalogar } \\
\text { No hay que estar } \\
\text { habituado a usar } \\
\text { SIGB } \\
\text { Muy fácil de usar }\end{array}$ & $\begin{array}{l}\text { Nivel de informática } \\
\text { básico } \\
\text { Nivel medio-alto de } \\
\text { inglés } \\
\text { Conocimientos avan- } \\
\text { zados de cataloga- } \\
\text { ción } \\
\text { Estar habituado a } \\
\text { usar SIGB } \\
\text { Complicado por falta } \\
\text { de guía }\end{array}$ & $\begin{array}{l}\text { Nivel de informática } \\
\text { básico } \\
\text { Conocimientos avan- } \\
\text { zados de cataloga- } \\
\text { ción } \\
\text { Estar habituado a } \\
\text { usar SIGB }\end{array}$ \\
\hline Actualización & $\begin{array}{l}\text { Sin fecha } \\
\text { Gratuita y sencilla }\end{array}$ & $\begin{array}{l}\text { Con fecha } \\
\text { Gratuita y sencilla }\end{array}$ & $\begin{array}{l}\text { Sin fecha } \\
\text { No se mencionan } \\
\text { otras versiones }\end{array}$ \\
\hline
\end{tabular}


A continuación se muestran imágenes de la interfaz principal de Biblio, BiblioteQ y CaMPI (en este mismo orden):

Interfaz principal del SIGB Biblio.

Imagen de elaboración propia, retocando la captura de imagen del programa Biblio.

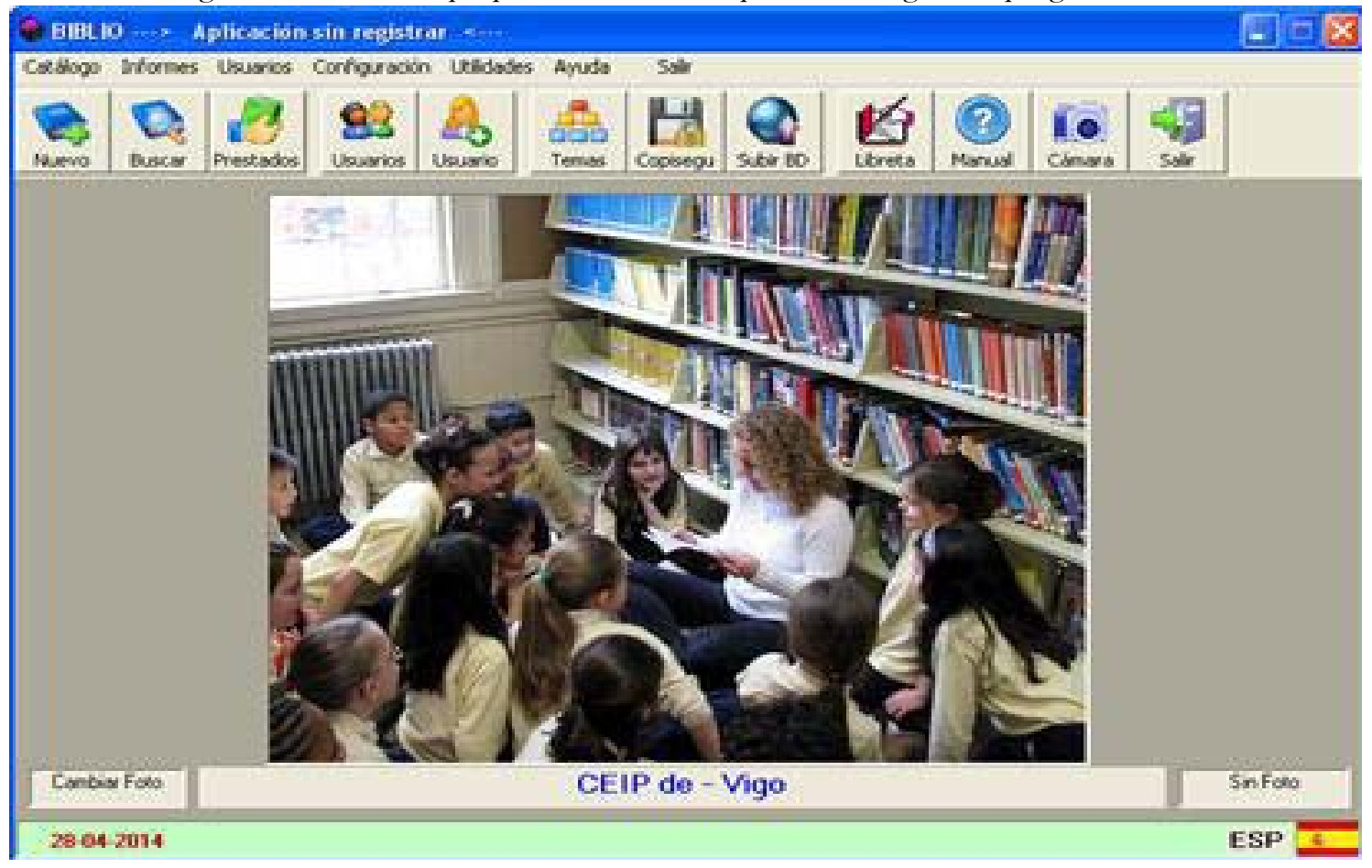

Interfaz principal del SIGB BiblioteQ.

Imagen de elaboración propia, retocando la captura de imagen del programa BiblioteQ.

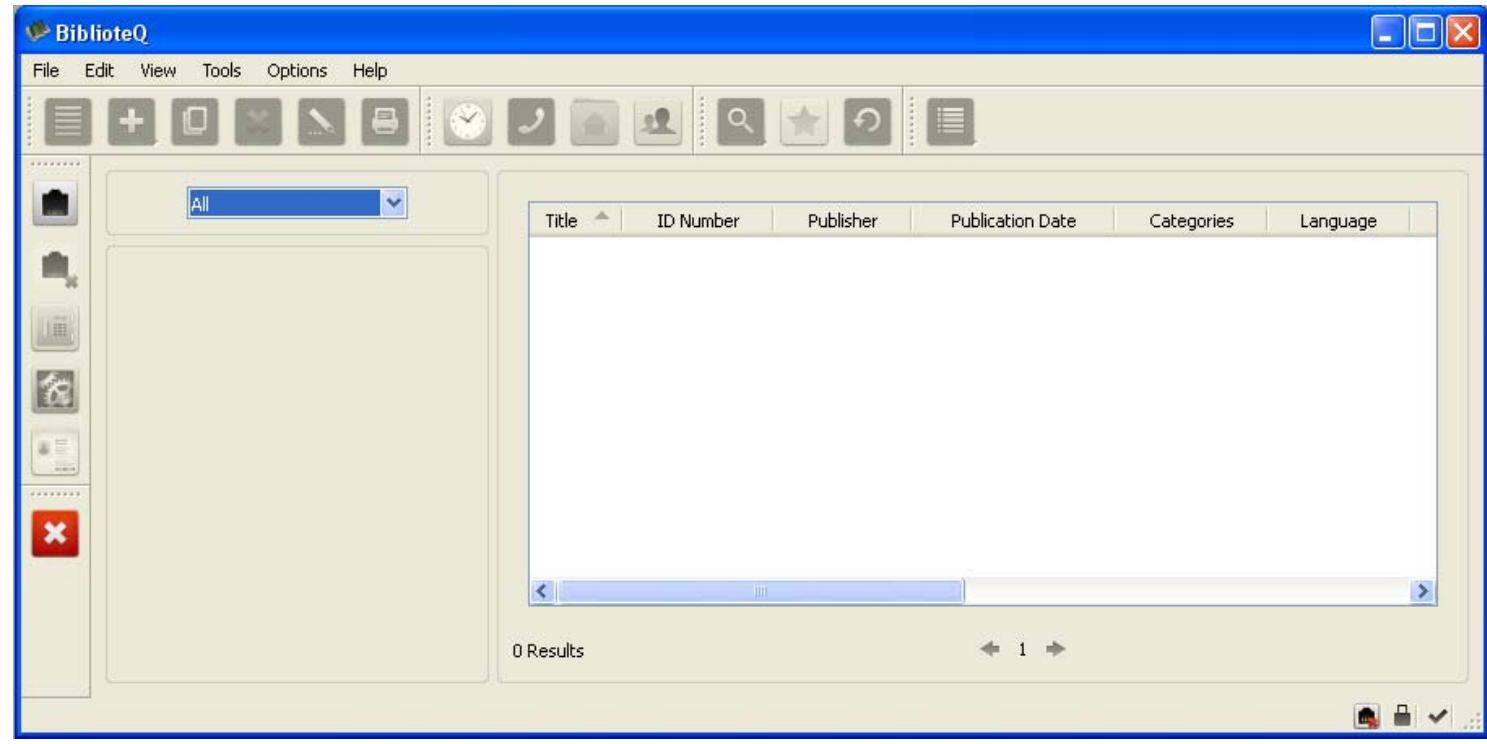




\section{Circulación}

\section{Administración}

\section{Estadisticas}

Catalogación

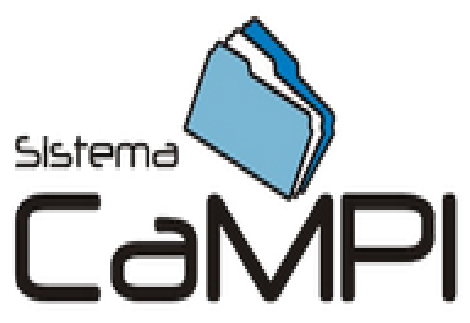

Opacmarc

\section{Sitio Web}

Una vez utilizados los tres programas, se compararon entre sí respecto a los módulos de gestión bibliotecaria en la tabla de elaboración propia que se presenta a continuación. Se indica con una "X" que el módulo está disponible en el programa, y con un “-” cuando no lo está.

\begin{tabular}{|c|c|c|c|}
\hline Módulo & Biblio & BiblioteQ & CaMPI \\
\hline Módulo de adquisiciones & - & $X$ & $X$ \\
\hline Módulo de catalogación & $\mathrm{X}$ & $\mathrm{X}$ & $\mathrm{X}$ \\
\hline $\begin{array}{c}\text { Módulo de control de } \\
\text { autoridades }\end{array}$ & - & - & $\mathrm{X}$ \\
\hline $\begin{array}{c}\text { Módulo de recuperación de } \\
\text { información }\end{array}$ & $\mathrm{X}$ & $\mathrm{X}$ & $\mathrm{X}$ \\
\hline Módulo de circulación & $\mathrm{X}$ & $\mathrm{X}$ & $\mathrm{X}$ \\
\hline $\begin{array}{c}\text { Módulo de publicaciones } \\
\text { periódicas }\end{array}$ & - & - & \\
\hline Módulo de estadísticas & $\mathrm{X}$ & $\mathrm{X}$ & $\mathrm{X}$ \\
\hline $\begin{array}{c}\text { Módulo de administración y } \\
\text { gestión }\end{array}$ & $\mathrm{X}$ & $\mathrm{X}$ & $\mathrm{X}$ \\
\hline
\end{tabular}


El sistema que cuenta con más módulos es CaMPI, con 7 de los 8 posibles, seguido de BiblioteQ, con 6 de 8, que respecto al anterior carece de módulo de control de autoridades. El menos completo es Biblio, con sólo 5 de 8 , ya al contrario de BiblioteQ, no dispone del módulo de adquisiciones. El módulo de publicaciones periódicas no cuenta con un módulo diferenciado de los demás en ninguno de los programas, pero en todos se permite realizar sus funciones.

De todos modos, Biblio es el único programa de los tres que no ha mostrado ningún mensaje de error ni ha impedido ejecutar alguna función, además de ser con diferencia el más sencillo de utilizar. Por lo tanto, que contenga menos módulos de gestión bibliotecaria en comparación con BiblioteQ y CaMPI no implica que no pueda suponer una solución válida para automatizar una biblioteca de pequeño volumen o particular. Como se menciona en la introducción, en la automatización de bibliotecas lo fundamental es acotar las necesidades del centro y buscar un sistema que se adapte a ellas.

\section{4.- CONCLUSIONES}

La estrategia de búsqueda dio lugar a una amplia lista de programas mencionados, presuntamente todos de acceso abierto y con los módulos necesarios para poder ser adecuadamente considerados SIGB. En internet figuran las reglas teóricas que debe de cumplir un software para ser calificado como libre y qué características teóricas debe abarcar un programa para ser considerado como SIGB. Sin embargo, con la verificación del listado se ha constatado que muchos de los programas que se mencionaban no se encontraban disponibles en la red, no eran realmente SIGB ni tampoco de acceso abierto. La experiencia de búsqueda ha demostrado por ejemplo que, en la práctica, la diferenciación entre software de acceso abierto y propietario no está realmente tan clara por la comunidad internauta. También se ha apreciado gran confusión respecto a lo que es un SIGB y los módulos que debe comprender para serlo.

La mayoría de SIGB libres estaban disponibles en el sitio web en forma de código descargable, que se obtenía mediante un simple click. Esto hacía presuponer un proceso de instalación fácil, pero el problema es que una vez descargado el código, hay que saber manejarlo apropiadamente. Otros programas venían comprimidos en archivos, que una vez abiertos no contaban con un archivo ejecutable. Finalmente estaban los que sí contaban con un archivo ejecutable, pero que necesitaban de otros programas externos y no 
proporcionados para su instalación o funcionamiento. Además, fue bastante complicado hallar guías de usuario o instrucciones de algún tipo que facilitaran estas instalaciones. Es decir, el acceso a estos programas es sencillo, y una vez instalados su manejabilidad es asumible por usuarios con un nivel informático medio, pero el proceso de instalación e implementación requiere unos conocimientos informáticos avanzados.

Los programas seleccionados, es decir, Biblio, BiblioteQ y CaMPI no eran los SIGB de acceso abierto más nombrados en la red, o los mejor considerados por los usuarios, ni tampoco los que contaban con funcionalidades más completas o profesionales. Pero sí que ofrecían al usuario un proceso de instalación sencillo, que a su vez facilitaba acceder a su utilización.

BiblioteQ y CaMPI eran mucho más completos y, naturalmente, más complejos de manejar. Compartían su perfil profesional y la amplia variedad de módulos disponibles. Ambos requerían que el usuario estuviese previamente acostumbrado a catalogar y a manejar SIGB. En ambos se permitía la captura de registros mediante el protocolo Z39.50. La principal diferencia entre ambos reside en que CaMPI necesita de conexión a internet y del navegador para funcionar. Se trata de programas muy potentes y profesionales capaces de gestionar grandes volúmenes de información, y pueden aportar una solución muy válida a las necesidades de automatización de diversos tipos centros con un gran volumen de información.

Biblio se diferencia de BiblioteQ y de CaMPI principalmente por requerir menos conocimientos para su uso. Se trata de un sistema más sencillo y ya anticuado, que en consecuencia necesitaría mejorarse. Puede ser una opción para bibliotecas particulares o pequeñas bibliotecas escolares.

Esta generación de software de licencia abierta y/o de acceso abierto no tiene nada que envidiar a sus hermanos privados en cuanto a funcionalidad, profesionalidad o versatilidad. Son igualmente capaces de llevar a cabo todas las tareas necesarias en una biblioteca actual con la rapidez, fiabilidad y precisión que deben ser siempre exigidas.

Los SIGB de acceso abierto llevan relativamente poco tiempo en el terreno de juego y su uso, pese a estar bastante extendido, aún tiene un largo camino por recorrer mientras sigue perfeccionándose. Esta perspectiva de futuro resulta muy beneficiosa en el mundo de la actualización de las bibliotecas porque cada vez habrá más opciones, y uno siempre puede elegir. 


\section{5.- BIBLIOGRAFÍA}

BREEDING, M., 2010. New models, core systems: discovery interfaces add a new facet to the market place: Automation Marketplace 2010. En: Library Journal [en línea]. Mayo/Junio 2011, volumen 47, número 4. Estados Unidos. [Consulta: 16 febrero 2014]. Disponible en: http://lj.libraryjournal.com/2010/04/library-services/automationmarketplace-2010-new-models-core-systems/

BREEDING, M. y YELTON, A., 2011. Librarians' assessment of automation systems". En: American Library Association [en línea]. Estados Unidos. [Consulta: 3 febrero 2014]. Disponible en: http://site.ebrary.com/lib/universvaln/docDetail.action? $\operatorname{docID}=10587963$

CLAYTON, M., 1991. Gestión automatizada de bibliotecas. Salamanca, Fundación Germán Sánchez Ruipérez.

ENIS, M., 2014. Open Source Options: Library Systems Landscape. En Library Journal [en línea]. Estados Unidos. [Consulta: 20 abril 2014]. Disponible en: http:// www.thedigitalshift.com/2014/04/ils/open-source-options-library-systems-landscape/

FUNDACIÓN CONTEMPORÁNEA, 2013. Séptima consulta semestral. Diciembre 2012. Análisis de resultados. En: Observatorio de la cultura [en línea]. Madrid. [Consulta: 30 enero 2014]. Disponible en: http://www.fundacioncontemporanea.com/ pdf/ANALISIS_RESULTÃDOS_OBS_DICIEMBRE_2012.pdf

GARCÍA MELERO, L.A. y GARCÍA CAMARERO, E., 1999. Automatización de bibliotecas. Madrid: Arco-Libros.

KENDALL, K. y KENDALL, J., 2005. Análisis y diseño de sistemas. México: Pearson Educación.

MINISTERIO DE EDUCACIÓN, CULTURA Y DEPORTE, 2006. Financiación y gasto público en cultura. En: Estadística 2006: Anuario de estadísticas culturales 2006 [en línea]. Madrid. [Consulta: 27 enero 2014]. Disponible en: http:// www.calameo.com/read/0000753355d6efb4a59c1

MINISTERIO DE EDUCACIÓN, CULTURA Y DEPORTE, 2013. Financiación y gasto público en cultura. En: Estadística 2013: Anuario de estadísticas culturales [en línea]. Madrid. [Consulta: 27 enero 2014]. Disponible en: http://www.mcu.es/ estadisticas/docs/capitulos_graficos/AEC2013/AEC_2013.pdf

MOYA, F., 1995. Los sistemas integrados de gestión bibliotecaria. Madrid: ANABAD.

MÜLLER, T. 2008. Étude comparative des principaux SIGB libres. [en línea] En: Premier congrès mondial de l'Association Internationale Francophone des Bibliothécaires Documentalistes (AIFBD) et colloque satellite IFLA 2008 en collaboration avec le Programme ALP «Francophonies et bibliothèques: innovations, changements et réseautage». Grande Bibliothèque, Bibliothèque et Archives nationales du Québec, Montréal. [Consulta: 16 febrero 2014]. Disponible en: http:// eprints.rclis.org/15389/

REYNOLDS, D. 1989. Automatización de bibliotecas: problemática y soluciones. Salamanca: Fundación Germán Sánchez Ruipérez

STALLMAN, R. 2004. Software libre para una sociedad libre [en línea]. GNU. [Consulta: 16 febrero 2014]. Disponible en: http://www.gnu.org/philosophy/fsfs/ free_software.es.pdf

TEDD, LA. 1988. Introducción a los sistemas automatizados de bibliotecas. Madrid: Díaz de Santos.

\section{6.- NOTAS}

1. Tutor del TFG, Adolfo Alonso Arroyo 